\title{
The psychobiology of nicotine dependence
}

\author{
D.J.K. Balfour
}

ABSTRACT: There is abundant evidence to show that nicotine is the principal addictive component of tobacco smoke. The results of laboratory studies have shown that nicotine has many of the behavioural and neurobiological properties of a drug of dependence.

This article focuses on the evidence that nicotine has the rewarding and reinforcing properties typical of an addictive drug and that these properties are mediated, in part, by its effects on mesolimbic dopamine neurones. However, in many experimental models of dependence, nicotine has relatively weak reinforcing properties that do not appear to explain adequately the powerful addiction to tobacco smoke experienced by many habitual smokers. Some of the reasons for this conundrum will be covered herein. This article focuses on the hypothesis that sensory stimuli and other pharmacologically active components in tobacco smoke play a pivotal role in the addiction to nicotine when it is inhaled in tobacco smoke.

The article will discuss the evidence that dependence upon tobacco smoke reflects a complex interaction between nicotine and the components of the smoke, which are mediated by complementary effects of nicotine on the dopamine projections to the shell and core subdivisions of the accumbens.

It will also discuss the extent to which the complexity of the dependence explains why nicotine replacement therapy does not provide a completely satisfying aid to smoking cessation and speculate on the properties treatments should exhibit if they are to provide a better treatment for tobacco dependence than those currently available.

KEYWORDS: Conditioned stimuli, laboratory models, mesolimbic dopamine, nicotine, reinforcement, tobacco dependence

$\mathrm{t}$ is now widely accepted that nicotine is the principal addictive component of tobacco smoke and that a majority of habitual smokers become dependent upon the nicotine they inhale. As a result, most smokers find it difficult to readily quit the habit in spite of the fact that they know that continuing to smoke is likely to cause significant harm to their health. Most countries in the developed world now seek to diminish the harm caused by tobacco by supporting major programmes directed at promoting smoking cessation. Many of these programmes advocate the use of nicotine replacement therapy (NRT) as an aid to smoking cessation. However, these programmes are often somewhat less successful than anticipated. This observation suggests that the addiction to tobacco may be more complex than a simple dependence upon nicotine. Preclinical studies in experimental animals have recently thrown significant light on the reasons why NRT may not provide a complete treatment for the addiction. This article will first consider the evidence that although nicotine has many of the properties of a drug of dependence, its reinforcing properties do not appear to be sufficiently strong enough to explain the powerful addiction to tobacco experienced by many habitual smokers. Some of the factors that may explain this conundrum will then be considered.

\section{THE ADDICTIVE PROPERTIES OF NICOTINE} Behavioural evidence that nicotine has

In the laboratory, the rewarding properties of nicotine have been investigated principally using two behavioural paradigms, conditioned place preference (CPP) and nicotine self-administration. In the CPP paradigm, injections of nicotine are paired with exposure to a specific compartment of an apparatus composed of two clearly distinguishable compartments; injections of the saline vehicle are paired with the other compartment. After a period of training, the animals are given free access to both compartments and the period spent in each compartment is compared. During this trial, if a drug has rewarding properties the animals spend more time in the compartment paired with the drug. Most of the major classes of drugs abused by humans serve as rewards in this procedure. However, experiments with nicotine using this paradigm have generated inconsistent results. There are a number of reports that nicotine does rewarding properties
CORRESPONDENCE

D.J.K. Balfour

Centre for Neuroscience

University of Dundee Medical School Ninewells Hospital

Dundee DD1 9SY UK

Fax: 441382633923

E-mail: d.j.k.balfour@dundee.ac.uk

STATEMENT OF INTEREST

D.J.K. Balfour has received an honorarium of US $\$ 2,500$ for speaking at a symposium organised by Pfizer, a fee of US $\$ 2,500$ for serving on a grant panel funded by Pfizer and a consultancy fee from Sanofi-Aventis for attending a research meeting. Research sponsorship was also received from Rothmans UK for two PhD students and a Postdoctoral researcher for approximately 7 years, this support ceased around 1990. D.J.K. Balfour's current work on nicotine and tobacco dependence is supported by grants from the Wellcome Trust and Cancer Research UK

European Respiratory Review Print ISSN 0905-9180 Online ISSN 1600-0617 
serve as a convincing reward in this procedure although it is often necessary to pair nicotine with the less preferred compartment of the apparatus in order to observe a clear response to the drug [1]. By contrast studies with experimental mice have provided more convincing evidence for a rewarding effect of nicotine [2]. Thus, the efficacy of this procedure seems to depend, to a significant extent, upon the species and, perhaps, the strain selected for the investigation. Nevertheless, these experiments have provided some evidence that nicotine has the rewarding properties which might be expected from a drug of dependence, although they seem to be less powerful and to yield less consistent results than those generated by experiments with other addictive drugs, such as cocaine, amphetamine or heroin.

The CPP paradigm allows researchers to investigate the rewarding properties of drugs. However, the drug is given non-contingently by the experimenter and is not under the control of the animal receiving the drug. Thus, the procedure does not lend itself to the investigation of the psychological and neurobiological mechanisms that mediate drug-seeking and drug-taking behaviour and characterise addiction. Selfadministration experiments, in which the animals are trained to perform a task in order to receive small doses of the drug, commonly delivered through an i.v. catheter (intravenous selfadministration; IVSA), provide a far better means of investigating these important aspects of addiction. Early studies in the 1980s demonstrated that nicotine could serve as a reinforcer in self-administration studies with monkeys and rats [3]. However, CORRIGALL and COEN [4] were among the first to report evidence that nicotine could serve as a robust reinforcer of IVSA in experimental rats. Subsequent studies in a number of laboratories $[5,6]$ have also demonstrated robust nicotine self-administration in rats. These studies have collectively opened the way for experiments designed to explore the psychobiological mechanisms that underpin the reinforcing properties of nicotine.

\section{The effects of nicotine withdrawal}

Many habitual smokers who try to quit the habit experience significant withdrawal symptoms during the early stages of abstinence and there is evidence that these symptoms contribute to relapse [7]. This observation provides one of the principal arguments for employing NRT as an aid to smoking cessation. A number of attempts have been made to model the effects of nicotine withdrawal in experimental animals. Perhaps the best established model is that first described by MALIN et al. [8]. In this experimental paradigm, the animals are constantly infused with nicotine delivered from a small subcutaneous mini-pump for periods ranging $7-$ 28 days. The abrupt withdrawal of nicotine generates a behavioural syndrome which MALIN et al. [8] argue models important components of the abstinence syndrome experienced by abstinent smokers. The behavioural response to nicotine withdrawal can also be precipitated by the administration of drugs that serve as antagonists of the neuronal nicotinic acetylcholine receptors (nAChR) through which nicotine exerts its effects [9]. This observation implies that the withdrawal effects reflect a neuroadaptation to sustained exposure to the drug, which are evoked when the response to nicotine is removed by either withdrawing the drug or antagonising its effects on the receptors. The response to nicotine withdrawal can be attenuated by the acute administration of nicotine using a protocol that seeks to model the effects of NRT [8]. Thus, the protocol would appear to have face validity as a model for the nicotine abstinence syndrome and has since been widely employed in many studies designed to investigate the neurobiological mechanisms that underpin the nicotine abstinence syndrome.

However, it is important to remember that the protocol used to evoke the abstinence syndrome in experimental animals does not seek to accurately model the development of dependence as it is experienced by human smokers. In the experimental model, the drug is delivered at a relatively high dose by constant infusion over a relatively short time. Furthermore, the behavioural characteristics observed following withdrawal closely resemble the effects of opiate withdrawal in this species [10]. The putative role of opiate mechanisms in nicotine dependence remains a matter of debate, although there is certainly no consensus at this time that they play a major role [11]. In recent years, PATERSON et al. [12] have explored the extent to which the nicotine abstinence syndrome can be observed in rats trained to self-administer the drug. They have shown that nicotine withdrawal following self-administration 7 days per week elicits spontaneous signs of abstinence when the drug is withdrawn, whereas its withdrawal following more limited availability ( $1 \mathrm{~h}$ per day for 5 days per week) does not result in the expression of spontaneous abstinence syndrome [12]. Thus, it seems reasonable to conclude that the overt behavioural signs of withdrawal reported by MALIN and co-workers $[8,10]$ are the product of neurobiological changes associate with sustained and substantial exposure to nicotine.

MALIN et al. [10] have argued that the effects of nicotine withdrawal they observed were caused, to some extent at least, by neuroadaptations to nicotine within the brain. However, another study has argued that a majority of the behavioural symptoms observed following the withdrawal of nicotine in this model are mediated peripherally [13]. In contrast, other studies have reported that the withdrawal of nicotine, following a period of sustained exposure, elicits an effect, an increase in brain reward threshold, which is clearly central in origin [14, 15]. The increase in brain reward threshold is investigated using electrodes located in one of the principal reward pathways of the brain, commonly the lateral hypothalamus. Increases in brain reward threshold are determined by measuring changes in the minimum current required to maintain self-stimulation of the electrodes. Nicotine withdrawal, as with the withdrawal of other psychostimulant drugs of dependence, increases this threshold. The change is thought to model the anhedonia (a reduced ability to respond for pleasurable stimuli) experienced by many smokers when they first quit [14].

\section{NEUROBIOLOGICAL MECHANISMS UNDERPINNING NICOTINE DEPENDENCE}

\section{Neural pathways implicated in nicotine dependence}

Nicotine exerts complex effects on many neural pathways within the brain by stimulating a family of neuronal nicotinic receptors. It seems likely that the neural mechanisms that underpin nicotine dependence reflect the complexity of these responses. A detailed discussion of the controversies surrounding these complexities lies beyond the scope of this 
article. Therefore, this article will focus upon one or two of the major theories concerning the neural basis of nicotine dependence. A majority of drugs dependence, especially those with psychostimulant properties broadly similar to those of nicotine, stimulate or enhance the release of dopamine (DA) in the principal terminal field of the mesolimbic system, the nucleus accumbens, and there is a consensus that the properties of these drugs play a central role in the neurobiology, underlying their potential to cause dependence [16, 17]. Studies in a number of laboratories have shown that nicotine injections also stimulate DA overflow in this area of the brain [11, 18-20]. Furthermore, lesions of the pathway attenuate substantially response for nicotine in an IVSA task [21]. The pathway projects to the nucleus accumbens from the ventral tegmental area in the midbrain. Micro-injections of the nAChR antagonist, dihydro- $\beta$-erythroidine, into this area of the brain also attenuate response for nicotine [22]. Other studies suggest that systemic injections of nicotine increase DA overflow in the nucleus accumbens by stimulating $\mathrm{nAChRs}$ on or close to the DA cell bodies in the ventral tegmental area [23-25]. Thus, it seems reasonable to conclude that the reinforcing properties of nicotine, measured using the IVSA paradigm, depend upon the increase in DA overflow evoked by stimulation of the DA neurones which project to the nucleus accumbens.

Less attention has been paid to the neurobiological mechanisms that underpin the effects of nicotine withdrawal. HildEBRAND et al. [26] reported that nicotine withdrawal, precipitated by the administration of a nicotinic receptor antagonist, is associated with reduced DA overflow in the nucleus accumbens and have suggested that this effect may mediate the anhedonia associated with nicotine withdrawal. More recent studies suggest that neuroadaptation of neurotransmission through mGluR2 and $\alpha$-amino-3-hydroxy-5methyl-isoxazole/kainate glutamatergic receptors located on DA neurones in the ventral tegmental area or on glutamate terminals, which innervate these DA neurones, play an important role in mediating the increase in brain reward function and, putatively, the anhedonia seen in nicotine withdrawn rats $[27,28]$.

Studies in a number of laboratories have shown that the effects of nicotine on mesolimbic DA neurones are complex and depend upon a number of factors, such as prior exposure to nicotine and the contingency with which the nicotine is delivered (i.e. is it administered noncontingently by the experimenter or is it administered upon the animal making a response, such as lever-pressing response in an IVSA paradigm). It is also clear that nicotine exerts differential effects on the neurones that project into the two principal subdivisions on the nucleus accumbens, the accumbens core and the accumbens shell. Experiments employing microdialysis probes, which sample the extracellular concentrations of DA in the interstitial space between the cells, have shown that acute non-contingent injections of nicotine preferentially or selective increase DA in the accumbal shell while having little or no effect on the projections to the accumbal core [29, 30]. However, daily injections of the drug for a few days result in a selective sensitisation of the DA projections to the accumbal core and, in these animals, subsequent non-contingent injections of nicotine cause a substantial and sustained increase in DA overflow. Daily non-contingent injections of other drugs of abuse also result in a regionally selective sensitisation of their effects on DA overflow in the accumbal core [31].

The DA response to self-administered nicotine is somewhat different. During the first few days of training, self-administered nicotine also preferentially increases DA overflow in the accumbal shell [32]. As the animals acquire the response (e.g. by week 3), there is evidence of a response in the accumbal core. However, in contrast to the effects of non-contingent nicotine, 3 weeks of training in an IVSA paradigm also results in sensitisation of its effects on DA overflow in the accumbal shell. Again, the self-administration of other drugs of dependence, such as cocaine or morphine, results in the sensitisation of their effects on DA overflow in the accumbal shell. Thus, the effects of nicotine on DA overflow in the nucleus accumbens resemble those seen in response to other drugs of abuse, and it seems reasonable to suggest that the effects are important to the mechanisms underlying the addictive potential of the drug. Mesolimbic DA neurones are innervated from a number of areas of the brain, notably, but not exclusively, the prefrontal cortex, hippocampus, amygdala and pedunculopontine tegmentum, which also form part of the limbic system and are likely to contribute to the mechanisms that mediate the neuroadaptive responses to repeated or chronic exposure to drugs of abuse [17, 33, 34]. It seems likely that changes in the activity of one or more of these projections, evoked by environmental stimuli that signal the availability of nicotine, modify the magnitude of the mesolimbic DA responses to the self-administered drug.

\section{THE PUTATIVE ROLE OF MESOLIMBIC DA IN ADDICTIVE BEHAVIOUR}

This article had previously highlighted the evidence that mesolimbic DA neurones appear to play important roles in both the reinforcing and rewarding properties of nicotine. However, it has become increasingly clear that the relationship between the effects of nicotine and its withdrawal on behaviour considered relevant to nicotine dependence are more complex than originally thought. There is evidence that the accumbal shell forms part of an extended amygdala and clearly forms part of the limbic system, whereas the accumbal core sends major projections to motor areas of the brain and may be associated more with the control of motor function [35, 36]. Thus, it seems reasonable to suggest that the effects of nicotine on DA overflow in the shell subdivision of the accumbens mediate its rewarding and reinforcing properties, whereas its effects on the DA projections to the accumbal core mediate the locomotor stimulant properties of the drug, which are also enhanced by prior exposure to the drug [18]. This explanation seems consistent with the behavioural data summarised above and with results generated by studies with other drugs, such as amphetamine and methylphenidate [37, 38]. However, it is important to note that the results of some experiments are not entirely consistent with this conclusion. For example, LAVIOLETTE and co-workers [39, 40] have reported that micro-injections of nicotine into the ventral tegmental area can elicit an aversive response to nicotine when measured using a conditioned place aversion task. Additionally, there is evidence that the administration of $N$-methyl-D-aspartate receptor antagonists can block both the development and expression of sensitised DA responses to nicotine measured in 
the accumbal core, whereas these antagonists do not influence the development or expression of the sensitisation locomotor response to nicotine $[41,42]$. Thus, the effects of nicotine on the DA projections to the accumbens do not invariably evoke a rewarding response nor are its effects on DA overflow in the accumbal core invariably tightly associated with increased locomotor activity. Therefore, any hypothesis that seeks to explain the role of mesolimbic DA in nicotine dependence needs to address these controversies.

The DA neurones that project to the nucleus accumbens can fire in different modes, for example as single irregular spikes or as bursts of activity, which are thought to encode different functions and have different consequences [43]. Furthermore, the electron microscope study of NIRENBERG et al. [44] suggests that the DA fibres which innervate the nucleus accumbens form two types of varicosity. Some form tight-synaptic contacts with neurones or dendrites within the accumbens. Others form "open synapses" that release DA directly into the interstitial space between the cells sampled by microdialysis probes. BALFOUR et al. [45] have suggested that single spikes of activity result in a preferential release of DA into the synaptic cleft. This results in activation of post-synaptic DA receptors. BALFOUR et al. [45] speculated that the DA released from the varicosities that form open synapses occurs preferentially when the neurones switch to the burst firing mode and diffuses into the interstitial space between the cells. By a process of volume transmission, this DA activates receptors on a number of adjacent cells. If this hypothesis is true, it seems reasonable to propose that DA released in response to single spikes may mediate events encoded by the phasic release of DA into the synaptic cleft, whereas DA released by burst firing may serve a paracrine function encoding more tonic events [18, 45]. Thus, for example, it may be possible to explain why both the aversive and reinforcing properties of nicotine may be associated with DA release from the DA neurones, which project to the nucleus accumbens, if it is hypothesised that the effects that the drug exerts on phasic and tonic DA release in different subdivisions of the accumbens subserve different functions and have different behavioural sequelae. This conclusion is largely consistent with the hypothesis proposed by GRACE et al. [33], who proposed that synaptic DA mediates events dependent upon the phasic release of DA whereas the DA in the interstitial space between the fibres mediates responses dependent upon the tonic release of DA. However, GRACE et al. [33] attribute different functions to burst and spike firing of the neurones. GRACE et al. [33] argue that burst firing of the neurones selectively stimulates DA release into the synaptic cleft whereas the tonic release of DA into the interstitial space is mediated by spike firing of the neurones.

One feature that is shared by psychostimulant drugs of abuse, such as amphetamine, cocaine and nicotine, is their ability to evoke a substantial and sustained increase in the concentration of DA in the extracellular space between the cells sampled directly using microdialysis probes. BALFOUR [11, 18] has argued that it is this shared property which confers addictive potential upon the drugs, and has speculated on the role increased extracellular DA in the accumbal core and shell might play in the neurobiology underlying addiction. There is a substantial body of evidence to support the hypothesis that the DA projections to the nucleus accumbens shell play a central role in the rewarding and reinforcing properties of these drugs when assessed using either the conditioned place preference paradigm of IVSA experiments [11, 18]. BALFOUR [11] has argued that the changes in the extracellular (putatively paracrine) DA concentration evoked by drugs of dependence in this subdivision of the accumbens are too sustained to be involved in encoding discrete events, such as the presentation of a single discrete reward. It was postulated that the primary role of this DA is to influence the probability that the individual, be it an experimental animal or a smoker, repeats specific behaviours associated with the delivery of a reward. Its primary psychophysiological role is to facilitate the acquisition of behaviours that result in the delivery of rewards, such as food when the individual is hungry. Studies with experimental animals support this hypothesis to the extent that they have shown the delivery of such rewards results in increased DA overflow in the accumbal shell [46]. Both DI ChiARA [19, 20] and BALFour $[11,18]$ have postulated that drugs of abuse exert such large and sustained effects on DA release in the shell that behaviours leading to the delivery of the drug become compulsive and, thereby, dominate the behavioural repertoire of an addicted individual. This implies that an addicted individual comes to derive great pleasure from the behaviour itself through its repeated association with increased DA overflow in the accumbal shell. The hypothesis may explain why, when delivery of the drug is dependent upon the behaviour, the effects of the drug on DA overflow in the accumbal shell are magnified further via conditioning [32, 47]. If the hypothesis is true, it represents an important component of the neurobiology underpinning the development of dependence.

It seems likely that the DA projections to the accumbal core play a role in the mediating elements of the locomotor stimulant properties of psychostimulant drugs [37, 38]. However, the locomotor stimulant properties of nicotine do not appear to be associated directly with changes in the extracellular DA concentration in this region of the brain since pharmacological interventions that block the effects of nicotine on DA overflow in the accumbal core do not invariably result in any changes in the locomotor response to the drug $[41,42,48]$. BALFOUR $[11,18]$ has proposed that extracellular DA in this subdivision of the accumbens plays a similar role to that proposed for extracellular DA in the accumbal shell, in that it serves to influence the probability that an individual will exhibit drug-seeking behaviour. However, its primary role appears to be to mediate the effects of sensory and environmental cues on drug-seeking behaviour, although the evidence to date that supports this conclusion is largely circumstantial. Nevertheless, ROBINSON and BERRIDGE $[49,50]$ have long argued that the sensitised DA responses evoked by the repetitive administration of addictive drugs influences the incentive salience of cues associated with delivery of the drugs. Furthermore, there is evidence from studies with cocaine that the non-contingent presentation of such a cue leads to both drug-seeking behaviour and a regionally selective increased DA overflow in the accumbal core $[51,52]$. Thus, it seems reasonable to hypothesise that the effects of repeated nicotine exposure on DA overflow in the accumbal shell and core exert complementary effects on nicotine-seeking behaviour which, together, serve to enhance greatly the addictive properties of the drug [11]. 


\section{THE ROLE OF CONDITIONED STIMULI IN NICOTINE DEPENDENCE}

Although nicotine appears to have the behavioural and neurobiological properties of a drug of dependence, its reinforcing properties in experimental rats are relatively weak when compared with those of many other drugs of abuse and do not appear to provide an adequate explanation for the powerful addictive properties of tobacco smoke [11,53]. There are a number of reasons why nicotine may not be a powerful reinforcer in this species. LE FolL et al. [54] have reported data which suggest that nicotine is more reinforcing in higher animals, such as monkeys, with complex cognitive skills, which are lacking in rodents. There is also evidence that tobacco smoke contains other, non-nicotinic compounds, which interact pharmacologically to enhance the neurobiological and behavioural responses to nicotine. For example, tobacco smoke contains compounds that inhibit the enzyme, monoamine oxidase, which metabolises DA and could, therefore, be expected to enhance the responses to nicotine mediated through DA [55]. There is, indeed, experimental evidence using animal models that inhibition of monoamine oxidase enhances the reinforcing properties of nicotine when measured using the IVSA paradigm [56].

Another important factor may be the fact that prolonged or sustained exposure to nicotine causes desensitisation of many of the nAChRs, which mediate its effects in the brain [11, 45]. As a result, while the administration of nicotine to animals with low circulating levels of nicotine results in stimulation of nAChRs, its administration to animals with blood nicotine concentrations similar to those found in the plasma of habitual smokers for much of the day fails to stimulate many of DA neurones in the ventral tegmental area or result in the release of DA in the nucleus accumbens $[57,58]$. BALFOUR and coworkers [11, 18, 25, 45] have argued that the relatively limited reinforcing properties of nicotine are related to the fact that sustained exposure to the drug causes desensitisation of nAChRs, thereby restricting the circumstances in which nicotine stimulates DA overflow in the nucleus accumbens. In this sense, the effects of nicotine contrast with those of other psychostimulant drugs of abuse which exert their effects on DA overflow through different molecular mechanisms and which persist for the full period of drug administration. Thus, any hypothesis that seeks to explain the role of nicotine in the addiction to tobacco must take into account the fact that, for many smokers, the inhalation of nicotine in tobacco may not necessarily result in the stimulation of the DA projections to the nucleus accumbens, which appears to be pivotal to the development of nicotine dependence.

In experimental models, the self-administration of psychostimulant drugs of abuse is facilitated significantly if delivery of the drug is paired with a sensory stimulus, such as a light and/or a tone. A series of studies by CAGGIULA and co-workers [59-61] have shown that the incorporation of this type of stimulus also greatly facilitates the acquisition and maintenance of nicotine self-administration. Indeed, in studies with experimental rats it often proves difficult to demonstrate significant levels of nicotine self-administration without using these stimuli. Importantly, once the association between a stimulus and nicotine delivery has been established, responding can be maintained, albeit at a lower level, by these stimuli in the absence of nicotine [59]. Furthermore, in rats in which responding for nicotine has been extinguished by replacing the drug reinforcer with an injection of saline, the presentation of the conditioned stimulus evokes nicotine-seeking behaviour $[53,59,61,62]$. This paradigm is commonly used to model the way in which cues associated delivery of an addictive drug precipitate relapse in an abstinent individual. Thus, these experiments suggest that conditioned stimuli can play an important role in nicotine self-administration in experimental animals.

Studies performed in human smokers imply that they probably play an equally important role in the addiction to tobacco. For example, Rose et al. [63] have reported that, in established smokers, sensory-motor cues present in tobacco smoke play a role in regulating the tobacco smoking habit and ameliorating the craving to smoke experienced by abstinent smokers. A more recent study showed that many habitual smokers found nicotine-free cigarettes almost as satisfying as regular cigarettes and that inhaling the smoke from these denicotinised cigarettes relieved the craving to smoke [64]. The sensory stimuli present in tobacco smoke seem to be particularly important in highly addicted smokers [65]. It seems reasonable to propose, therefore, that the powerful addiction to tobacco reflects an interaction between a relatively weak reinforcing drug, nicotine, and the sensory cues present in the tobaccosmoke vehicle in which it is delivered $[18,66]$.

The nature of the interaction between the sensory motor cues associated with the delivery of nicotine and "satisfaction" derived by the smoker when they inhale tobacco smoke may be more complex than was initially thought. Sensory motor cues may serve as "classical" conditioned stimuli in which their efficacy as conditioned reinforcers depends entirely upon their close association with behaviours that deliver the drug (e.g. smoking in humans, lever-pressing in rats trained to respond for nicotine). In the absence of such conditioning, these stimuli do not have any intrinsic reinforcing properties. Recent experiments have provided evidence that association with the delivery of nicotine can confer reinforcing properties on stimuli of this type [53,62]. However, nicotine also appears to have the ability to significantly enhance the reinforcing properties of an otherwise weakly reinforcing stimulus. This has been demonstrated using a paradigm in which rats were trained to switch off the house light in an operant chamber (rats prefer low light environments). In this paradigm, control animals can be trained press a lever to extinguish the light, although this reward does not have powerful reinforcing properties [61]. In rats treated with nicotine, responding for this stimulus is greatly enhanced. However, DoNNY et al. [61] showed that the effect of nicotine in this paradigm does not depend upon a close association between a self-administered i.v. injection of the drug and the presentation of the stimulus. Indeed, in a yoked design, animals who received the nicotine injections non-contingently (i.e. they did not control when they received the injections) pressed just as often as the rats in which presentation of the sensory stimulus was tightly associated with the contingent self-administration of nicotine. These data imply that the rats were responding primarily for the sensory stimulus (extinguishing the house light) and that nicotine greatly enhanced the reinforcing properties of the 
stimulus irrespective on the dependency with which it was delivered.

In the study by DONNY et al. [61], small i.v. injections of nicotine were delivered throughout the 1-h trials. A recent pilot study in the current authors' laboratory has demonstrated that a single subcutaneous injection of the drug, delivered $10 \mathrm{~min}$ before the trial, exerts a very similar effect on responding reinforced by extinguishing the house light (fig. 1). These results clearly support those of DONNY et al. [61], but also suggest that a single injection of nicotine exerts effects in the brain that persist long enough to facilitate responding for the stimulus for at $1 \mathrm{~h}$. Nicotine exerts a number of effects in the brain which could mediate this effect. Interestingly, however, the non-contingent administration of amphetamine, at a dose that elicits a sustained increase in DA overflow in the nucleus accumbens, facilitates responding for a conditioned stimulus previously paired with another reinforcer $[67,68]$. Therefore, it seems reasonable to speculate that the sustained effects that nicotine exerts on DA overflow in the accumbens may also mediate its effects on responding for a weak reinforcer; however, other possible explanations should not be excluded.

\section{THE IMPLICATIONS OF LABORATORY STUDIES FOR OUR UNDERSTANDING OF PSYCHOPHARMACOLOGY AND TREATMENT OF TOBACCO DEPENDENCE}

The laboratory studies summarised above have shown that nicotine has behavioural and neurobiological properties characteristic of a drug of dependence and provide clear support for the primary role of nicotine in the addiction to tobacco. Thus, they also provide support for the use of NRTs as an aid to smoking cessation. NRT clearly elicits a significant and clinically valuable increase in the number of smokers who are able to successfully quit their habit $[69,70]$. However, the pharmacology underlying the effectiveness of this treatment may be more complex than a simple substitution therapy. A majority of NRT preparations deliver nicotine to the brain more slowly than cigarette smoke and it seems plausible that the main therapeutic effect of NRTs reflects their ability to maintain plasma nicotine levels at a sufficiently high concentration to limit the development of nicotine withdrawal [11]. More recently, varenicline, a drug that acts as a partial agonist at the $\alpha_{2} \beta_{4} \mathrm{nAChR}$, has been introduced as an aid to smoking cessation [71]. In many ways, this compound mimics the pharmacological properties which are seen as being important to addiction, i.e. the ability to both stimulate and block the $\alpha_{2} \beta_{4}$ $n A C h R$. At the experimental level, the administration of this compound to drug-free experimental rats increases DA overflow in the nucleus accumbens but blocks the effects of a subsequent challenge with nicotine [72]. Furthermore, it inhibits nicotine self-administration and attenuates the reinstatement of nicotine-seeking behaviour evoked by a noncontingent injection of nicotine [72]. Thus, it might be expected to limit the effects of nicotine withdrawal while at the same time preventing a challenge dose of nicotine exerting effects in the brain which mediate reinforcement. The hypothesis would seem to hold true to the extent that the drug is clearly an effective aid to smoking cessation with an efficacy at least as great, and possibly greater than, NRT [73].

Another non-nicotinic approach that has proved valuable is bupropion. The efficacy of bupropion as an aid to smoking cessation was discovered serendipitously and the psychobiology underlying its efficacy in this indication remains the subject of debate. This compound, or its active metabolites, potentiate the effects of norepinephrine and DA in the brain by blocking the transporters which re-accumulate these neurotransmitters into the nerve terminals, and it is assumed that this effect is important to its mechanism of action as an aid to smoking cessation [74]. If this is true, it seems reasonable to hypothesise that the compound works by alleviating some of the consequences of nicotine/ tobacco withdrawal through its effects on these pathways, perhaps most notably the anhedonia experienced by many abstinent smokers. Recent studies with experimental animals provided some support for this hypothesis, to the extent that the
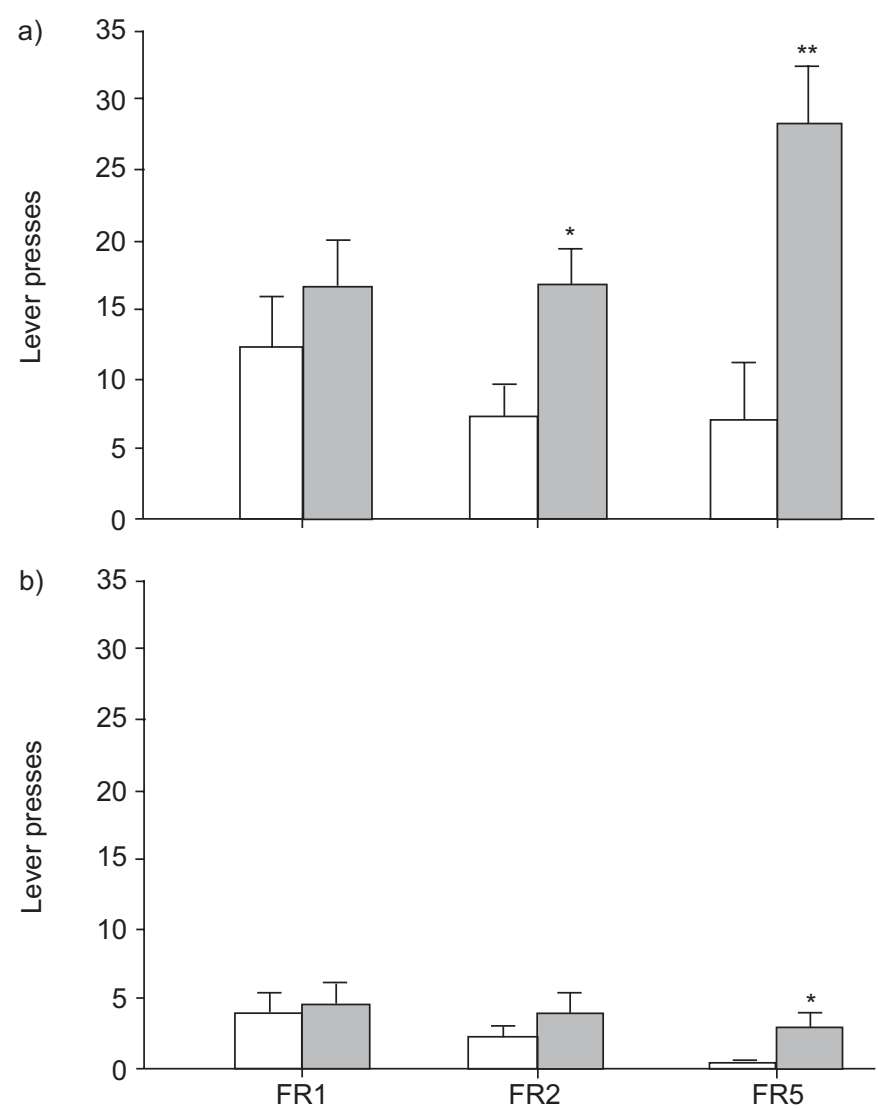

FIGURE 1. The effects of non-contingent nicotine on responding for a weak reinforcer. Responses in an operant chamber on a) the active lever, which turned out the house light for $20 \mathrm{~s}$, and $\mathrm{b}$ ) the inactive lever, which had no programmed consequences. The rats were given a subcutaneous injection of saline ( $\square$ ) or nicotine $\left(\square ; 0.4 \mathrm{mg} \cdot \mathrm{kg}^{-1}\right) 10 \mathrm{~min}$ before being placed in the operant chamber. Responses were measured over the course of the next 60 min. Response was measure on a fixed ratio (FR) schedule of reinforcement with a 20-s time-out between the availability of the reward. Data are reported for responding on an FR1, FR2 and FR5 schedule in which the rats were required to press once, twice or five times for the reinforcer. Pressing on the active lever was significantly higher than the inactive lever $(F(1,20)=28.1, p<0.001)$. In the rats treated with nicotine but not saline, the increase in response seen on the active lever as the FR increased was significant $(F(2,20)=9.4 ; p<0.001)$. This was significantly different from rats treated with saline. Data are expressed as mean \pm SEM of six observations. *: $p<0.05$; **: $p<0.01$. (N. Montgomery and D.J.K. Balfour; unpublished data) 


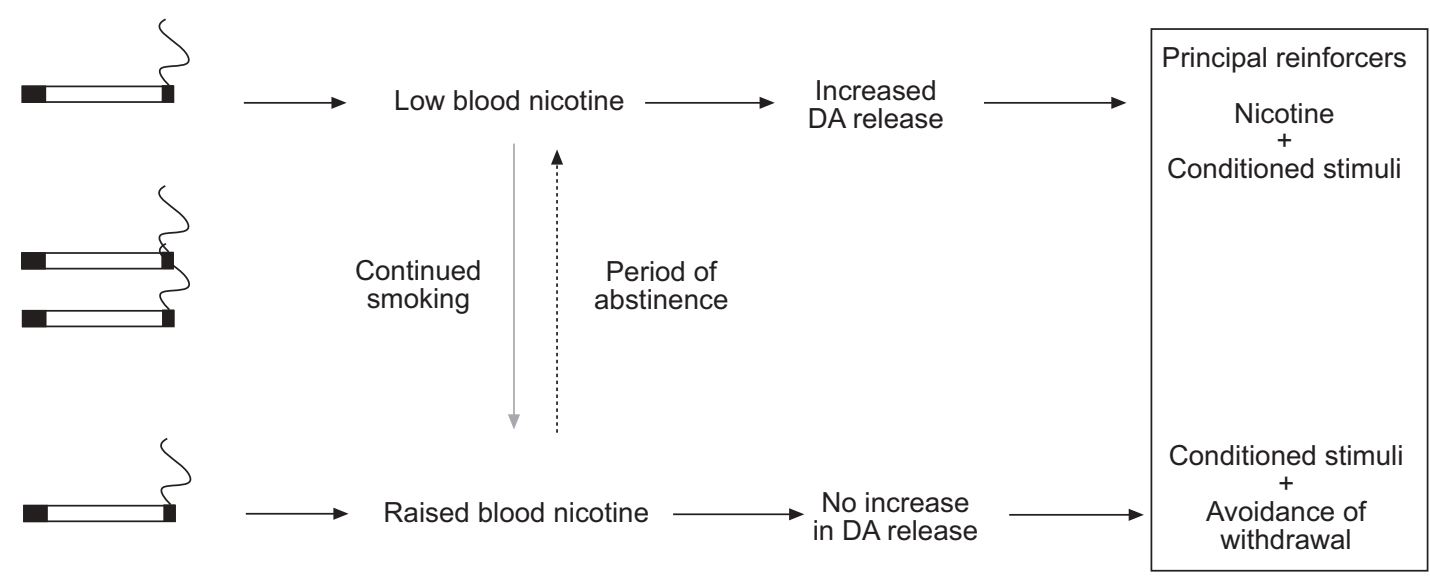

FIGURE 2. Tobacco smoking as a second order schedule of reinforcement. Schematic diagram showing the postulated differential dopamine (DA) responses to nicotine in tobacco smoke when delivered following a period of abstinence and following a period of continued smoking. This causes an accumulation of nicotine in the blood and brain resulting in desensitisation of neuronal nicotinic receptors. The figure demonstrates the proposed role of nicotine, conditioned sensory stimuli within tobacco smoke and avoidance of withdrawal as reinforcers of the tobacco smoking habit in nicotine-abstinent and nicotine-replete individuals.

compound attenuates the anhedonia measured in nicotine abstinent rats [75]. Bupropion also attenuates the somatic consequences of nicotine withdrawal [76]. Interestingly, in animals allowed to self-administer nicotine, bupropion did not reduce responses for the drug although it did diminish the increase in brain reward function exhibited by these animals, suggesting a dissociation between these two responses to the drug [76].

Although the use of NRT results in a significant and clinically valuable increase in the number of smokers who are able to quit successfully, this approach is often not as successful as therapists and smokers would like. The results of the studies with experimental animals have, perhaps, provided an explanation for this conundrum. The experimental data suggest that nicotine may not be an intrinsically powerful reinforcer when compared with many of the other drugs of abuse used by humans. This article has summarised some of the reasons why this may be true and considered some of the reasons why a drug that is a relatively weak reinforcer may, nevertheless, be of fundamental importance to the powerful addiction to tobacco smoke experienced by many smokers. It is possible that the addiction reflects an interaction between the reinforcing properties of the drug and the complex cognitive skills of humans relative to the experimental animals in which most of the behavioural studies have been performed [54]. However, it also now seems likely that the sensory stimuli within the tobacco smoke vehicle are also of primary importance and the addiction to tobacco reflects a psychobiological interaction between these stimuli and the effects of nicotine in the brain [11]. Thus, for many smokers the dependence upon tobacco may usefully be equated to a second order schedule of reinforcement in which the reinforcing properties of nicotine per se are experienced relatively infrequently. For the periods when the nAChRs are desensitised, which may be significant parts of the day, the habit is reinforced by cues and conditioned stimuli present in the smoke [11]. The hypothesis is summarised in figure 2. This possibility impacts significantly on the approaches that need to be adopted to treat tobacco dependence more successfully than it is at present.

It seems reasonable to propose that nicotine-based therapies preferentially target the nicotinic component of the addiction. This is probably also true of other therapies, such as varenicline, which depend upon their effect on nicotinic systems within the brain. Drugs which antagonise these receptors have been shown to attenuate the rewarding properties of nicotine, measured using the CPP paradigm $[77,78]$, and the reinforcing properties of the drug assessed using nicotine IVSA [79, 80]. Interestingly, however, the administration of CB1 receptor antagonists attenuates the persistent nicotine-seeking behaviour reinforced by the presentation of conditioned stimuli previously associated with the delivery of nicotine [62] and the reinstatement of nicotineseeking behaviour evoked by the non-contingent presentation of a priming dose of nicotine or a conditioned stimulus [80]. These observations support the conclusion that endocannabinoid systems within the brain play a role in the neurobiology underpinning nicotine dependence and suggest that drugs targeting the CB1 receptors which mediate their effects may be useful in the treatment of tobacco dependence. Importantly, these compounds seem to target a system putatively implicated in the mechanisms that mediate the role of conditioned stimuli in the addiction [81].

In summary, therefore, the evidence that nicotine is the primary addictive component of tobacco remains robust. However, studies in recent years suggest that the tobacco smoke vehicle in which it is delivered also plays a pivotal role in the addiction to tobacco. Tobacco smoke contains other pharmacologically active agents that can enhance or facilitate the dependence upon nicotine. Additionally, it would seem that the dependence upon nicotine is facilitated by the close association with sensory cues within the smoke, which both greatly enhance nicotine-seeking behaviour and can, themselves, maintain the smoking habit. Future improvements in the treatment for tobacco dependence and relapse will need to address this component of the addiction. 


\section{REFERENCES}

1 Le Foll B, Goldberg SR. Nicotine induces conditioned place preferences over a large range of doses in rats. Psychopharmacology 2005; 178: 481-492.

2 Grabus SD, Martin BR, Brown SE, Damaj MI. Nicotine place preference in the mouse: influences of prior handling, dose and strain and attenuation by nicotinic receptor antagonists. Psychopharmacology 2006; 184: 456-463.

3 Goldberg SR, Spealman RD, Goldberg DM. Persistent behavior at high rates maintained by intravenous selfadministration of nicotine. Science 1981; 214: 573-575.

4 Corrigall WA, Coen KM. Nicotine maintains robust selfadministration in rats on a limited access schedule. Psychopharmacology 1989; 99: 473-478.

5 Donny EC, Caggiula AR, Knopf S, Brown C. Nicotine selfadministration in rats. Psychopharmacology 1995; 122: 390-394.

6 Shoaib M, Schindler CW, Goldberg SR. Nicotine selfadministration in rats: strain and nicotine pre-exposure effects on acquisition. Psychopharmacology 1997; 129: 35-43.

7 Hughes JR. Effects of abstinence from tobacco: etiology, animal models, epidemiology, and significance: a subjective review. Nicotine Tob Res 2007; 9: 329-339.

8 Malin DH, Lake JR, Newlin-Maultsby P, et al. Rodent model of nicotine abstinence syndrome. Pharmacol Biochem Behav 1992; 43: 779-784.

9 Malin DH, Lake JR, Carter VA, et al. The nicotinic antagonist mecamylamine precipitates nicotine abstinence syndrome. Psychopharmacology 1994; 115: 339-342.

10 Malin DH. Nicotine dependence studies with a laboratory model. Pharmacol Biochem Behav 2001; 70: 551-559.

11 Balfour DJK. Complementary roles for the accumbal shell and core in nicotine dependence. In: Bock G, Goode J, eds. Understanding Nicotine and Tobacco Addiction. Novartis Foundation. Chichester, John Wiley \& Sons, 2006; pp 96-115.

12 Paterson NE, Markou A. Prolonged nicotine dependence associated with extended access to nicotine self-administration in rats. Psychopharmacology 2004; 173: 64-72.

13 Watkins SS, Stinus L, Koob GF, Markou A. Reward and somatic changes during precipitated nicotine withdrawal in rats; centrally and peripherally mediated effects. J Pharmacol Exp Ther 2000; 292: 1053-1064.

14 Epping-Jordan MP, Watkins SS, Koob GF, Markou A. Dramatic decreases in brain reward function during nicotine withdrawal. Nature 1998; 393: 76-79.

15 Kenny PJ, Markou A. Neurobiology of the nicotine withdrawal syndrome. Pharmacol Biochem Behav 2001; 70: 531-549.

16 Di Chiara G. Nucleus accumbens medial shell and core dopamine: differential role in behavior and addiction. Behav Brain Res 2002; 137: 75-114.

17 Vanderschuren LJ, Kalivas PW. Alterations in dopaminergic and glutamatergic transmission in the induction and expression of behavioral sensitization: a critical review of preclinical studies. Psychopharmacology 2000; 151: 99-120.

18 Balfour DJK. The neurobiology of tobacco dependence: a preclinical perspective on the role of the nucleus accumbens. Nicotine Tob Res 2004; 6: 899-912.

19 Di Chiara G. Role of dopamine in the behavioural actions of nicotine related to addiction. Eur J Pharmacol 2000; 393: 295-314.
20 Di Chiara G. Behavioural pharmacology and neurobiology of nicotine reward and dependence. In: C. Clementi, D. Fornasari, C. Gotti, eds. Handbook of Experimental Pharmacology. Vol. 14. Berlin, Springer, 2000; pp. 603-750.

21 Corrigall WA, Franklin KJB, Coen KM, Clarke PBS. The mesolimbic dopaminergic system is implicated in the reinforcing effects of nicotine. Psychopharmacology 1992; 107: 285-289.

22 Corrigall WA, Coen KM, Adamson KL. Self-administered nicotine activates the mesolimbic dopamine system through the ventral tegmental area. Brain Res 1994; 653: 278-284.

23 Benwell MEM, Balfour DJK, Lucchi HM. Influence of tetrodotoxin and calcium on changes in extracellular dopamine levels evoked by systemic nicotine. Psychopharmacology 1993; 112: 467-474.

24 Nisell M, Nomikos GG, Svensson TH. Systemic nicotineinduced dopamine release in the rat nucleus accumbens is regulated by nicotinic receptors in the ventral tegmental area. Synapse 1994; 16: 36-44.

25 Balfour DJK, Benwell MEM, Birrell CE, Kelly RJ, AlAloul M. Sensitization of the mesoaccumbens dopamine response to nicotine. Pharmacol Biochem Behav 1998; 59: 1021-1030.

26 Hildebrand BE, Nomikos GG, Hertel P, Schilström B, Svensson TH. Reduced dopamine output in the nucleus accumbens but not the prefrontal cortex in rats displaying mecamylamine-precipitated nicotine withdrawal syndrome. Brain Res 1998; 779: 214-225.

27 Kenny PJ, Gasparini F, Markou A. Group II metabotropic and $\alpha$-amino-3-hydroxy-5-methyl-4-isoxazole propionate (AMPA)/kainate glutamate receptors regulate the deficit in brain reward function associated with nicotine withdrawal in rats. J Pharmacol Exp Ther 2003; 306: 1068-1076.

28 Markou A. Metabotropic glutamate receptor antagonists: novel therapeutics for nicotine dependence and depression? Biol Psychiat 2006; 61: 17-22.

29 Cadoni C, Di Chiara G. Differential changes in the accumbens medial shell and core dopamine in behavioural sensitization to nicotine. Eur J Pharmacol 2000; 387: R23-R25.

30 Iyaniwura TT, Wright AE, Balfour DJK. Evidence that mesoaccumbens dopamine and locomotor responses to nicotine in the rat are influenced by pre-treatment dose and strain. Psychopharmacology 2001; 158: 73-79.

31 Cadoni C, Solinas M, Di Chiara G. Psychostimulant sensitization: differential changes in accumbal medial shell and core dopamine. Eur J Pharmacol 2000; 388: 69-76.

32 Lecca D, Cacciapaglia F, Valentini V, Gronli J, Spiga S, Di Chiara G. Preferential increase of extracellular dopamine in the rat nucleus accumbens shell as compared to that in the core during acquisition and maintenance of intravenous nicotine self-administration. Psychopharmacology 2006; 184: 435-446.

33 Grace AA, Floresco SB, Goto Y, Lodge DJ. Regulation of firing of dopaminergic neurons and control of goaldirected behaviors. Trends Neurosci 2007; 30: 220-227.

34 Bardo MT. Neuropharmacological mechanisms of drug reward: beyond dopamine in the nucleus accumbens. Crit Rev Neurobiol 1998; 12: 37-67.

35 Heimer L, Zahm DS, Churchill L, Kalivas PW, Wohltman C. Specificity in the projection patterns of 
accumbal core and medial shell in the rat. Neuroscience 1991; 41: 89-125.

36 Zahm DS, Brog JS. On the significance of subterritories in the "accumbens" part of the rat ventral striatum. Neuroscience 1992; 50: 751-767.

37 Sellings LH, Clarke PB. Segregation of amphetamine reward and locomotor stimulation between nucleus accumbens medial medial shell and core. J Neurosci 2003; 23: 6295-6303.

38 Sellings LH, McQuade LE, Clarke PB. Characterization of dopamine-dependent rewarding and locomotor stimulant effects of intravenously-administered methylphenidate in rats. Neuroscience 2006; 141: 1457-1468.

39 Laviolette SR, Alexson TO, van der Krooy D. Lesions of the tegmental pedunculopontine nucleus block the rewarding effects and reveal the aversive effects of nicotine in the ventral tegmental area. J Neurosci 2002; 22: 8653-8660.

40 Laviolette SR, van der Krooy D. The neurobiology of nicotine addiction: bridging the gap from molecules to behaviour. Nat Rev Neurosci 2004; 5: 55-65.

41 Shoaib M, Benwell MEM, Akbar MT, Stolerman IP, Balfour DJK. Behavioural and neurochemical adaptations to nicotine in rats: influence of NMDA antagonists. $\mathrm{Br} J$ Pharmacol 1994; 111: 1073-1080.

42 Balfour DJ, Birrell CE, Moran RJ, Benwell ME. Effects of acute D-CPPene on mesoaccumbens dopamine responses to nicotine in the rat. Eur J Pharmacol 1996; 316: 153-156.

43 Schultz W. Behavioral theories and the neurophysiology of reward. Ann Rev Psychol 2006; 57: 87-115.

44 Nirenberg MJ, Chan J, Pohorille A, et al. The dopamine transporter: comparative ultrastructure of dopaminergic axons in limbic and motor compartments of the nucleus accumbens. J Neurosci 1997; 17: 6899-6907.

45 Balfour DJK, Wright AE, Benwell MEM, Birrell CE. The putative role of extra-synaptic mesolimbic dopamine in the neurobiology of nicotine dependence. Behav Brain Res 2000; 113: 73-83.

46 Bassareo V, Di Chiara G. Differential responsiveness of dopamine transmission to food-stimuli in nucleus accumbens shell/core compartments. Neuroscience 1999; 89: 637-641.

47 Lecca D, Cacciapaglia F, Valentini V, Acquas E, Di Chiara G. Differential neurochemical and behavioral adaptation to cocaine after response contingent and noncontingent exposure in the rat. Psychopharmacology 2007; 191: 653-667.

48 Benwell ME, Holtom PE, Moran RJ, Balfour DJ. Neurochemical and behavioural interactions between ibogaine and nicotine in the rat. Br J Pharmacol 1996; 117: 743-749.

49 Robinson TE, Berridge KC. The neural basis of drug craving: an incentive-sensitization theory of addiction. Brain Res Rev 1993; 18: 247-291.

50 Robinson TE, Berridge KC. Addiction. Ann Rev Psychol 2003; 54: 25-53.

51 Ito R, Dalley JW, Howes SR, Robbins TW, Everitt BJ. Dissociation in conditioned dopamine release in the nucleus accumbens core and medial shell in response to cocaine cues and during cocaine-seeking behaviour in rats. J Neurosci 2000; 20: 7489-7495.
52 Ito R, Robbins TW, Everitt BJ. Differential control over cocaine-seeking behaviour by nucleus accumbens core and shell. Nature Neurosci 2004; 7: 389-397.

53 Palmatier MI, Evans-Martin FF, Hoffman A, et al. Dissociating the primary and reinforcement-enhancing effects of nicotine using a rat self-administration paradigm with concurrently available drug. Psychopharmacology 2006; 184: 391-400.

54 Le Foll B, Wertheim C, Goldberg SR. High reinforcing efficacy of nicotine in non-human primates. PLoS One 2007; 2: e230.

55 Fowler JS, Logan J, Wang GJ, Volkow ND. Monoamine oxidase and cigarette smoking. Neurotoxicology 2003; 24: 75-82.

56 Guillem K, Vouillac C, Azar MR, et al. Monoamine oxidase A rather than monoamine oxidase $\mathrm{B}$ inhibition increases nicotine reinforcement in rats. Eur J Neurosci 2006; 24: 3532-3540.

57 Pidoplichko V, De Biasi M, Williams JT, Dani J. Nicotine activates and desensitizes midbrain dopamine neurones. Nature 1997; 390: 401-404.

58 Benwell MEM, Balfour DJK, Birrell CE. Desensitisation of nicotine-induced dopamine responses during constant infusion with nicotine. Br J Pharmacol 1995; 114: 211-217.

59 Caggiula AR, Donny EC, White AR, et al. Cue dependency of nicotine self-administration and smoking. Pharmacol Biochem Behav 2001; 70: 515-530.

60 Caggiula AR, Donny EC, Chaudhri N, Perkins KA, EvansMartin FF, Sved AF. Importance of nonpharmacological factors in nicotine self-administration. Physiol Behav 2002; 77: 683-687.

61 Donny EC, Chaudhri N, Caggiula AR, et al. Operant responding for a visual reinforcer in rats is enhanced by noncontingent nicotine: implications for nicotine selfadministration and reinforcement. Psychopharmacology 2003; 169: 68-76.

62 Cohen C, Perrault G, Griebel G, Soubrié P. Nicotineassociated cues maintain nicotine-seeking behavior in rats several weeks after nicotine withdrawal: reversal by the cannabinoid (CB1) receptor antagonist, rimonabant. Neuropsychopharmacology 2005; 30: 145-155.

63 Rose JE, Behm FM, Levin ED. Role of nicotine dose and sensory cues in the regulation of smoke intake. Pharmacol Biochem Behav 1993; 44: 891-900.

64 Rose JE, Behm FM, Westman EC, Johnson M. Dissociating nicotine and nonnicotine components of cigarette smoking. Pharmacol Biochem Behav 2000; 67: 71-81.

65 Brauer LH, Behm FM, Lane JD, Westman EC, Perkins C, Rose JE. Individual differences in smoking reward from de-nicotinized cigarettes. Nicotine Tob Res 2001; 3: 101-109.

66 Rose JE, Behm FM, Westman EC, Bates JE, Salley A. Pharmacologic and sensorimotor components of satiation in cigarette smoking. Pharmacol Biochem Behav 2003; 76: 243-250.

67 Taylor JR, Robbins TW. Enhanced behavioural control by conditioned reinforcers following microinjections of $\mathrm{d}$ amphetamine into the nucleus accumbens. Psychopharmacology 1984; 84: 405-412.

68 Wyvell CL, Berridge KC. Intra-accumbens amphetamine increases the pure incentive salience of a Pavlovian cue for 
food reward: enhancement of "wanting" without either "liking" or reinforcement. J Neurosci 2000; 20: 8122-8130.

69 Etter JF, Stapleton JA. Nicotine replacement therapy for long-term smoking cessation: a meta-analysis. Tob Cont 2006; 15: 280-285.

70 West $R$, Zhou X. Is nicotine replacement therapy for smoking cessation effective in the "real world"? Findings from a prospective multinational cohort study. Thorax 2007; 62: 998-1002.

71 Nides M. Update on pharmacologic options for smoking cessation treatment. Am J Med 2008; 121: Suppl. 1, S20-S31.

72 Rollema H, Chambers LK, Coe JW, et al. Pharmacological profile of the $\alpha 4 \beta 2$ nicotinic acetylcholine receptorpartial agonist varenicline, an effective smoking cessation aid. Neuropharmacology 2007; 52: 985-994.

73 Stapleton JA, Watson L, Spirling LI, et al. Varenicline in the routine treatment of tobacco dependence: a pre-post comparison with nicotine replacement therapy and an evaluation in those with mental illness. Addiction 2008; 103: 146-154.

74 Dwoskin LP, Rauhut AS, King-Pospisil KA, Bardo MT. Review of the pharmacology and clinical profile of bupropion, an antidepressant and tobacco use cessation agent. CNS Drug Rev 2006; 12: 178-207.
75 Paterson NE, Balfour DJ, Markou A. Chronic bupropion attenuated the anhedonic component of nicotine withdrawal in rats via inhibition of dopamine reuptake in the nucleus accumbens shell. Eur J Neurosci 2007; 25: 3099-3108.

76 Paterson NE, Balfour DJK, Markou A. Chronic bupropion differentially alters the reinforcing, reward-enhancing and conditioned motivational properties of nicotine in rats. Nicotine Tob Res 2008; 10: 995-1008.

77 Le Foll B, Goldberg SR. Rimonabant, a CB1 antagonist, blocks nicotine-conditioned place preferences. NeuroReport 2004; 15: 2139-2143.

78 Forget B, Hamon M, Thiébot M-H. Cannabinoid CB1 receptors are involved in motivational effects of nicotine in rats. Psychopharmacology 2005; 181: 722-734.

79 Cohen C, Perrault G, Voltz C, Steinberg R, Soubrié P. SR141716, a central cannabinoid (CB1) receptor antagonist, blocks the motivational and dopamine-releasing effects of nicotine. Behav Pharmacol 2002; 13: 451-463.

80 Shoaib M. The cannabinoid antagonist AM251 attenautes nicotine self-administration and nicotine-seeking behaviour in rats. Neuropharmacology 2008; 54: 438-444.

81 Cohen C, Kodas E, Griebel G. CB1 receptor antagonists for the treatment of nicotine addiction. Pharmacol Biochem Behav 2005; 81: 387-395. 